\title{
CARACTERIZAÇÃO MORFOLÓGICA DE FONTES DE RESISTÊNCIA DE MELOEIRO A Pseudoperonospora cubensis ${ }^{1}$
}

\author{
LEIDIANE BEZERRA ALBUQUERQUE ${ }^{2 *}$, RAFAELA PRISCILA ANTONIO ${ }^{3}$, GLAUBER HENRIQUE DE SOUSA \\ NUNES $^{4}$, RAVIER VALCÁCER DE MEDEIROS ${ }^{2}$, ANTONIO JOSÉ RODRIGUES DA SILVA FILHO ${ }^{4}$
}

\begin{abstract}
RESUMO - O míldio, causado pelo fungo Pseudoperonospora cubensis é uma das principais doenças foliares que acometem a cultura do meloeiro no período chuvoso da região Nordeste brasileira. O objetivo do presente trabalho foi identificar e caracterizar morfologicamente fontes de resistência de meloeiro a P. cubensis. Trinta e seis acessos e quatro cultivares comerciais foram avaliados em delineamento em blocos casualizados com três repetições. A unidade experimental foi composta por sete plantas. E a avaliação realizada em condições de campo com uma escala diagramática, a qual fora calculado o índice de porcentagem da doença (IPD). As fontes de resistência e genótipos referências foram caracterizadas morfologicamente. Os acessos C-RN-2, C-SE-2, CCE-2, C-PE-2, C-PI-1 e C-BA-2 apresentam resistência a $P$. cubensis e são promissores para uso em programas de melhoramento visando resistência ao míldio do meloeiro, embora os seus frutos tenham reduzida qualidade comercial.
\end{abstract}

Palavras-chave: Cucumis melo. Míldio. Germoplasma. Resistencia. Qualidade.

\section{MORPHOLOGICAL CHARACTERIZATION OF RESISTANCE MELON SOURCES TO Pseudoperonospora cubensis}

\begin{abstract}
The downy mildew, caused by the fungus Pseudoperonospora cubensis is a major foliar disease that attacks the melon crop in the rainy season in northeastern Brazil. The objective of this study was to identify and characterize morphologically resistant melon accessions to $P$. cubensis. Thirty-six accessions and four commercial cultivars were evaluated in a randomized block design with three replications. The plot was composed of seven plants. The evaluation was conducted in the under field conditions with a diagrammatic scale and calculating the disease percentage index (DPI). Resistance sources and references genotypes were characterized morphologically. The accessions C-RN-2, C-SE-2, C-CE-2, C-PE-2, C-PI-1, and C-BA-2 show resistance to $P$. cubensis and are promising for use in programs breeding for resistance to downy mildew although its fruits have reduced commercial quality.
\end{abstract}

Keywords: Cucumis melo. Downy mildew. Germplasm. Resistance. Quality.

\footnotetext{
*Autor para correspondência

${ }^{1}$ Recebido para publicação em 02/09/2014; aceito em 06/05/2015.

Parte da dissertação de mestrado do primeiro autor.

${ }^{2}$ Programa de pós-graduação em Fitotecnia, UFERSA, Caixa Postal 137, 59625-900, Mossoró (RN), leidy_albuquerque@hotmail.com, raviermedeiros@yahoo.com.br.

${ }^{3}$ Embrapa Semiárido, Caixa Postal 23, 56302 $\square 970$, Petrolina (PE), rafaela.antonio@embrapa.br.

${ }^{4}$ Departamento de Ciências Vegetais, UFERSA, Caixa Postal, 137, 59625-900, Mossoró (RN); glauber@ufersa.edu.br, feevida@hotmail.com.
} 


\section{INTRODUÇ̃̃̃}

O míldio, causado pelo oomiceto Pseudoperonospora cubensis [(Berkeley \& Curtis) Rostovzev, 1903] se destaca como uma das principais doenças foliares que acometem a cultura do meloeiro durante o período chuvoso na região Nordeste brasileira, chegando a causar perdas econômicas substanciais aos produtores dessa cultura (CARDOSO; SANTOS; VIDAL, 2002). Considerado um dos agentes patogênicos mais destrutivos da família Cucurbitaceae, $P$. cubensis possui uma ampla gama de hospedeiros, dentre os quais se destaca o melão como uma das espécies mais importantes do ponto de vista econômico (LEBEDA; WIDRLECHNER; URBAN, 2006).

O processo de patogênese do míldio está bem elucidado. Contudo, poucas opções de controle estão disponíveis para a referida doença (OLCZAKWOLTMAN; MARCINKOWSKA; NIEMIROWICZ-SZCZYTT, 2011). Uma das alternativas mais promissoras consiste no uso de cultivares resistentes, sendo esta uma estratégia de fácil adoção pelo agricultor, segura ao meio ambiente e complementar ao controle químico, reduzindo os custos de produção (COHEN; SCHREIBER; NERSON, 1995; URBAN; LEBEDA, 2007; THINES; VOGLMAYR; GÖKER, 2009). A identificação de fontes de resistência a um determinado patógeno consiste em uma das etapas iniciais para o desenvolvimento de um programa de melhoramento (CÂNDIDA et al., 2009). Para tanto, torna-se imprescindível a avaliação dos acessos no germoplasma com o objetivo de se identificar fontes de resistência promissoras.

A busca por alelos de resistência pode ser realizada mediante a avaliação de acessos existentes na agricultura tradicional. No caso do meloeiro, mesmo tendo seus centros de origem, domesticação primária e secundária em regiões distantes do Brasil, possui variedades tradicionais adaptadas às diferentes condições edafoclimáticas nacionais. Este germoplasma encontra-se em poder de pequenos agricultores que produzem, conservam e selecionam as próprias sementes a partir dos frutos que consideram de qualidade superior e as cultivam no plantio seguinte (QUEIROZ, 2004; NEITZKE et al., 2009). Mesmo de forma inconsciente, esses materiais foram selecionados por várias gerações, resultando em diversos genótipos de meloeiro rústicos e adaptados às condições locais onde foram selecionados.

São poucos os relatos sobre a reação do germoplasma de meloeiro a $P$. cubensis em razão da dificuldade de manutenção deste fungo biotrófico. Uma das primeiras avaliações foram realizadas nos
Estados Unidos e identificaram o acesso PI 124112 como altamente resistente, enquanto os acessos PI 124111, PI 122847, PI 124210, PI 145594 e PI 16552 foram resistentes (THOMAS, 1982; THOMAS; JOURDAIN, 1992). Avaliações posteriores em 1076 acessos identificaram como resistentes PI 271329 e PI 401644 (THOMAS, 1999). A linhagem MR-1 apresenta-se como resistente, sendo a herança devido a dois genes de efeitos complementares denominados Pc-1 e Pc-2 (THOMAS, 1986). No Brasil, foram avaliadas 28 cultivares comerciais em condições de campo. Embora tenha se observado variabilidade entre os genótipos todos foram suscetíveis (SANTOS et al., 2009). E não há informações sobre a reação do germoplasma nacional a $P$. cubensis, sendo, portanto, necessários esforços para identificar acessos resistentes.

A caracterização da variação das novas fontes de resistência é uma tarefa necessária em programas de melhoramento que visam a seleção de genótipos resistentes com alta produtividade e características qualitativas de valor comercial. A caracterização tem grande relevância por dois aspectos: o primeiro está relacionado com a própria preservação da variabilidade genética existente na agricultura tradicional. A intenção é manter essa variabilidade presente nas propriedades, conservando-a em bancos de germoplasma ou mesmo in loco, evitando, por conseguinte, a erosão genética pela introdução de cultivares melhoradas. Um segundo aspecto está relacionado com os programas de melhoramento genético, pois as variedades tradicionais são importantes, principalmente por se constituírem em fontes de alelos.

O objetivo do presente trabalho foi identificar e caracterizar morfologicamente fontes de resistência de meloeiro a $P$. cubensis coletadas em diferentes estados da região Nordeste do Brasil.

\section{MATERIAL E MÉTODOS}

\section{Germoplasma}

Foram avaliados 36 acessos de meloeiro, coletados em diferentes Estados da região Nordeste do Brasil (Tabela 1). Os acessos integram a coleção de germoplasma de cucurbitáceas da UFERSA, os quais foram coletados em plantas individuais e autofecundados por cinco gerações. Além disso, foram avaliadas quatro cultivares comerciais comumente utilizadas por produtores da região como 'Iracema', 'Olimpic', 'Amaral' e 'Mabel', todas suscetíveis a $P$. cubensis. 
L. B. ALBUQUERQUE et al.

Tabela 1. Origem e grupo botânico dos acessos e cultivares avaliados quanto à reação a $P$. cubensis em condições de campo.

\begin{tabular}{|c|c|c|c|c|c|}
\hline Acesso & Origem & Grupo botânico & Acesso & Origem & Grupo botânico \\
\hline C-MA-1 & Maranhão & momordica & C-PB-1 & Paraíba & Chate \\
\hline C-MA-2 & Maranhão & cantalupensis & C-PB-2 & Paraíba & Chate \\
\hline C-MA-3 & Maranhão & momordica & C-PE-1 & Pernambuco & Conomom \\
\hline C-MA-4 & Maranhão & momordica & C-PE-2 & Pernambuco & Conomom \\
\hline C-MA-5 & Maranhão & conomom & C-PE-3 & Pernambuco & cantalupensis \\
\hline C-PI-1 & Piauí & momordica & C-PE-4 & Pernambuco & cantalupensis \\
\hline C-PI-2 & Piauí & momordica & C-AL-1 & Alagoas & ND \\
\hline C-PI-3 & Piauí & momordica & C-AL-2 & Alagoas & Momordica \\
\hline C-CE-1 & Ceará & cantaloupensis & C-AL-3 & Alagoas & Momordica \\
\hline C-CE-2 & Ceará & momordica & C-SE-1 & Sergipe & Momordica \\
\hline C-CE-3 & Ceará & momordica & C-SE-2 & Sergipe & ND \\
\hline C-RN-1 & Rio G. Norte & cantaloupensis & C-BA-1 & Bahia & Acidulus \\
\hline C-RN-2 & Rio G. Norte & cantaloupensis & C-BA-2 & Bahia & Momordica \\
\hline C-RN-3 & Rio G. Norte & cantaloupensis & C-BA-3 & Bahia & Momordica \\
\hline C-RN-4 & Rio G. Norte & ND & C-BA-4 & Bahia & Momordica \\
\hline C-RN-5 & Rio G. Norte & cantaloupensis & C-BA-5 & Bahia & Momordica \\
\hline C-RN-6 & Rio G. Norte & momordica & 'Iracema' & Sakata ${ }^{\mathbb{B}}$ & Inodorus \\
\hline C-RN-7 & Rio G. Norte & momordica & 'Olimpic' & Sakata ${ }^{\circledR}$ & Reticulatus \\
\hline C-RN-8 & Rio G. Norte & momordica & 'Amaral' & Rijk Zwaan $^{\circledR}$ & Inodorus \\
\hline C-RN-9 & Rio G. Norte & momordica & 'Mabel' & Rijk Zwaan $^{\circledR}$ & Inodorus \\
\hline
\end{tabular}

ND: não definida. Classificação de acordo com Pitrat (2008).

\section{Condução experimental}

A pesquisa foi realizada entre os meses de fevereiro a abril de 2012 em condições de campo, sob infecção natural, na área experimental da Horta Didática da Universidade Federal Rural do SemiÁrido - UFERSA, em Mossoró (RN).

$\mathrm{O}$ experimento foi conduzido em blocos casualizados completos, com 40 tratamentos em três repetições. E a unidade experimental composta por uma linha de 3,5 metros, totalizando sete plantas por parcela.

As sementes dos acessos e cultivares foram semeadas em bandejas de poliestireno expandido com 128 células preenchidas com substrato comercial Plantmax ${ }^{\circledR}$. O transplantio das mudas para o campo foi realizado aos 15 dias após a semeadura, quando as mesmas estavam com um par de folhas definitivas. A cultura foi irrigada por gotejamento, com fertirrigação, no espaçamento de 2,0 m entre linhas e $0,5 \mathrm{~m}$ entre gotejadores. As fitas de gotejamento utilizadas eram de $16 \mathrm{~mm}$, com vazão de 1,6 L/h. As demais práticas culturais e o manejo seguiram as recomendações para a cultura no Estado (NUNES et al., 2005).

A avaliação da severidade da doença foi realizada em campo, aos 24 dias após o transplantio. Foram selecionadas aleatoriamente cinco plantas de cada parcela, com 15 folhas aleatórias por planta e atribuídas notas com auxílio de uma escala diagramática proposta por Michereff et al. (2009), com modificações. Com os ajustes, a escala passou a ser composta por notas que variaram de zero a cinco, onde: zero - sem sintoma; 1 - menos de 10 pontos isolados na área foliar; 2 - 11 a 20 pontos isolados na área foliar; 3 - manchas e 30\% da área foliar afetada; 4 - manchas necróticas e $50 \%$ da área foliar afetada; e 5 - manchas necróticas e mais de $50 \%$ da área foliar afetada.

Os dados das notas por parcela com a média das 75 folhas (cinco plantas com 15 folhas/planta) foram utilizados para o cálculo do índice de porcentagem de doença (IPD), de acordo com McKinney (1923), em que:

$\mathrm{IPD}=\frac{\sum \text { (grau da escala X frequência) }}{\text { (número total de unidades } \mathrm{X} \text { grau máximo da escala) }} \times 100$

De acordo com o valor do IPD foram propostas cinco categorias para agrupamento dos genótipos, quais sejam: (1) 0: altamente resistente (AR); (2) 112,99: resistente (R); (3) 13-25,99: moderadamente suscetível (MS); (4) 26-50,0: suscetível (S); e (5) > 50: altamente suscetível (AS).

\section{Análises estatísticas}

As análises foram realizadas com a média do índice de porcentagem de doença das parcelas com o modelo misto na forma matricial por $\mathrm{y}=\mathrm{Xr}+\mathrm{Zg}+$ e, em que y é o vetor de dados observados conhecidos, r é o vetor dos efeitos de blocos (assumidos como fixos), g é o vetor dos efeitos genotípicos desconhecidos (assumidos como aleatórios) e o vetor de erros (aleatórios) e $\mathrm{X}$ e $\mathrm{Z}$ matrizes de incidência para os referidos efeitos (RESENDE et al., 2002), sendo $g$ $\sim \mathrm{N}(0, \mathrm{G}), \mathrm{e} \sim \mathrm{N}(0, \mathrm{R}), \mathrm{E}(\mathrm{y})=\mathrm{Xr}$ e V(y) = ZGZ' + R. G é a matriz de variâncias ecovariâncias dos efeitos genéticos e R matriz de variâncias e covariâncias 
dos erros. Para esse modelo, as equações de modelos mistos são:

$$
\left(\begin{array}{cc}
X^{\prime} R^{-1} X & X^{\prime} R^{-1} Z \\
Z^{\prime} R^{-1} X & Z^{\prime} R^{-1}+G^{-1}
\end{array}\right)\left(\begin{array}{l}
\hat{r} \\
\hat{g}
\end{array}\right)=\left(\begin{array}{l}
X^{\prime} R^{-1} y \\
X^{\prime} R^{-1} y
\end{array}\right)
$$

As deviances foram obtidas rodando-se o modelo com e sem os valores de $\mathrm{h}^{2}$ (para acessos). Em seguida, subtraindo-os e confrontando-os com o valor do Qui-quadrado com um grau de liberdade a $5 \%$ de probabilidade. A obtenção de estimadores e preditores em modelos mistos foi realizada por processos iterativos. Foram obtidos os componentes de variância genotípíca e residual, herdabilidade média, acurácia seletiva, coeficiente de variação genético, coeficiente de variação ambiental e coeficiente de variação relativa.

\section{Caracterização morfológica}

A caracterização dos acessos foi realizada em um delineamento em blocos casualizados com três repetições. A unidade experimental correspondeu a uma linha composta por dez plantas no espaçamento $2,0 \times 0,3 \mathrm{~m}$. Os caracteres qualitativos utilizados foram cor do epicarpo, cor do mesocarpo, rachadura de fruto, expressão sexual, formato do fruto e abscisão do pedúnculo (IPGRI, 2003). As avaliações foram feitas pela observação visual das características. Os caracteres quantitativos avaliados foram massa do fruto (obtida em balança eletrônica com capacidade de $25,0 \mathrm{~kg}$ e precisão de $0,01 \mathrm{~g}$. O resultado foi expresso em kg), número de frutos por planta, espessura da polpa (medida utilizando um paquímetro digital e tomada na região equatorial, a partir dos frutos seccionados transversalmente, a parte do fruto que contém o início da cavidade placentária até o início da formação da casca), expressa em milímetros $(\mathrm{mm})$ e firmeza da polpa (medida após corte transversal do fruto, sendo tomadas três leituras por fruto em cada lado da polpa, na região equatorial, equidistantes em relação ao comprimento e à espessura da polpa, utilizando-se um penetrômetro modelo Wagner ${ }^{\circledR}$ com pluncher de ponta cônica de $8,0 \mathrm{~mm}$ (Fruit Pressure Tester - FT 011. MOD) para medir a resistência na polpa. Os resultados foram expressos em Newton $(\mathrm{N})$, sólidos solúveis (medido após a retirada uma amostra de aproximadamente $2 / 3$ da espessura da polpa na região equatorial do fruto, no sentido da cavidade), cuja amostra foi pressionada manualmente até que uma parte do suco fosse depositada em um refratômetro digital (Digital Refractometer Palette 100) e os resultados foram expressos em ${ }^{\circ}$ Brix).

\section{RESULTADOS E DISCUSSÃO}

Em qualquer avaliação de acessos ou seleção de genótipos superiores é fundamental que o ensaio tenha elevada precisão. A acurácia seletiva tem sido o parâmetro utilizado para avaliar a qualidade experimental por conter informações da magnitude da variação residual, do número de repetições e proporção entre as variações de natureza genética e residual associadas ao caráter em avaliação. A acurácia correlaciona o valor genotípico verdadeiro do tratamento genético com o predito a partir da informação fenotípica obtida no experimento e pode variar de 0 a $1(0$ a $100 \%$ ). O valor de acurácia seletiva encontrado nesta pesquisa foi de 0,88 (Tabela 2) e está associado a alta precisão do ensaio, de acordo com Resende e Duarte (2007).

Tabela 2. Análise de Deviance e estimativas de parâmetros do índice de porcentagem da doença (IPD) avaliado em acessos de meloeiro com P. cubensis em condições de campo.

\begin{tabular}{ccccccccc}
\hline Efeito/Parâmetros & \multicolumn{7}{c}{ Índice de porcentagem da doença (Estimativas) } \\
\cline { 2 - 8 } & Deviance & $\mathrm{V}_{\mathrm{GEN}}$ & $\mathrm{V}_{\mathrm{E}}$ & $\mathrm{h}_{\mathrm{m}}{ }_{\mathrm{m}}(\%)$ & $\mathrm{A}_{\mathrm{GEN}}(\%)$ & $\mathrm{CV}_{\mathrm{g}}(\%)$ & $\mathrm{CV}_{\mathrm{e}}(\%)$ & $\mathrm{CVr}(\%)$ \\
\hline Modelo completo & 691,48 & 85,44 & 75,29 & 77,30 & 0,88 & 40,66 & 38,17 & 1,57 \\
Efeito genotípico & 698,78 & & & & & & & \\
$\chi^{2}$ & $7,30^{* *}$ & & & & & & & \\
\hline
\end{tabular}

$\mathrm{V}_{\mathrm{GEN}}$ - variância genotípica, $\mathrm{V}_{\mathrm{E}}$ - variância residual, $\mathrm{h}_{\mathrm{m}}^{2}$ - herdabilidade média, $\mathrm{A}_{\mathrm{GEN}}$ - acurácia seletiva, $\mathrm{CV}_{\mathrm{g}}$ - coeficiente de variação genética, $\mathrm{CV}_{\mathrm{e}}$ - coeficiente de variação ambiental, $\mathrm{CVr}$ - coeficiente de variação relativa. ${ }^{* *}$ : Significativo $(\mathrm{p}<0,01)$ pelo teste de Qui-quadrado para efeito genotípico.

Outro aspecto fundamental em ensaios envolvendo a reação de genótipos a determinado patógeno em condições de campo é a uniformidade da distribuição do patógeno na área. No presente trabalho, verificou-se que o míldio foi bem distribuído em todos os blocos do experimento. Todas as parcelas das cultivares testemunhas suscetíveis e grande parte dos acessos apresentaram severidade semelhante nos diferentes blocos.

Os valores das deviances para o modelo completo e para o modelo sem o efeito de acessos (genotípico) estão na Tabela 2. Observou-se diferença significativa entre os acessos pelo teste de Quiquadrado $(\mathrm{p}<0,01)$, indicando variabilidade genética entre os acessos para o índice de porcentagem de doença.

A herdabilidade é um dos mais importantes parâmetros para a atividade do melhorista. $\mathrm{O}$ referido parâmetro quantifica quanto da variação fenotípica é devida a causas genéticas. A herdabilidade varia de 0 a 1,0 e quanto maior a sua estimativa mais segurança terá o melhorista no processo seletivo. $\mathrm{Na}$ presente 
pesquisa, a estimativa do coeficiente de herdabilidade é alta $(77,30 \%)$ e evidencia bom controle genético na expressão do caráter, indicando grande potencial para seleção dentro do experimento (Tabela 2). Outra medida importante é o coeficiente de variação relativa $\left(\mathrm{CV}_{\mathrm{g}} / \mathrm{CV}_{\mathrm{e}}\right)$. Conforme comenta Vencovsky (1987), valores acima da unidade indicam uma condição favorável para o processo seletivo, fato observado no presente trabalho.

Na Tabela 3 estão dispostos a ordem e o valor genotípico dos acessos para o índice de porcentagem de doença (IPD). Valores genotípicos devem ser os preferíveis pelos melhoristas, pois são estes os verdadeiros valores a serem preditos. Os acessos foram classificados em três classes de reação: resistente, moderadamente resistente e suscetível. Entre os acessos, $25 \%$ foram suscetíveis $(26,00 \leq$ IPD $\leq 50,00), 58,3 \%$ moderadamente resistentes $(13,00 \leq \mathrm{IPD} \leq 25,99)$ e $16,7 \%$ resistentes $(1,00 \leq$ IPD $\leq 12,99)$. Vale salientar que parte das cultivares comerciais utilizadas para produção nacional de meloeiro não são desenvolvidas nas condições ambientais das regiões produtoras de melão do Brasil (VARGAS et al., 2010),

Tabela 3. Ordenamento de acessos, valor genotípico $(\mathrm{u}+\mathrm{g})$ do índice de porcentagem da doença (IPD) e reação de acessos de meloeiro com P. cubensis em condições de campo.

\begin{tabular}{|c|c|c|c|c|c|c|c|}
\hline Acesso & Ord. & $\mathrm{u}+\mathrm{g}$ & Reação & Acesso & Ord. & $\mathrm{u}+\mathrm{g}$ & Reação \\
\hline C-RN-9 & 1 & 41,10 & $\mathrm{~S}$ & C-MA-4 & 21 & 23,73 & MR \\
\hline C-BA-4 & 2 & 36,90 & $\mathrm{~S}$ & C-AL-2 & 22 & 23,03 & MR \\
\hline C-PI-3 & 3 & 34,78 & $\mathrm{~S}$ & C-PB-1 & 23 & 21,65 & MR \\
\hline 'Amaral' & 4 & 34,33 & S & C-SE-1 & 24 & 20,48 & MR \\
\hline 'Olimpic' & 5 & 33,06 & $\mathrm{~S}$ & C-BA-3 & 25 & 20,00 & MR \\
\hline 'Iracema' & 6 & 33,06 & S & C-RN-7 & 26 & 19,07 & MR \\
\hline 'Mabel' & 7 & 32,30 & S & C-PI-2 & 27 & 19,01 & MR \\
\hline C-RN-8 & 8 & 30,33 & S & C-PB-2 & 28 & 18,77 & MR \\
\hline C-BA-1 & 9 & 30,31 & S & C-MA-1 & 29 & 18,18 & MR \\
\hline C-CE-3 & 10 & 27,83 & $\mathrm{~S}$ & C-PE-3 & 30 & 15,26 & MR \\
\hline C-BA-4 & 11 & 27,77 & S & C-RN-5 & 31 & 14,88 & MR \\
\hline C-RN-6 & 12 & 27,56 & S & C-MA-2 & 32 & 14,47 & MR \\
\hline C-PE-4 & 13 & 26,60 & $\mathrm{~S}$ & C-AL-1 & 33 & 13,78 & MR \\
\hline C-MA-3 & 14 & 25,08 & MR & C-RN-1 & 34 & 13,49 & MR \\
\hline C-AL-1 & 15 & 24,98 & MR & $\mathrm{C}-\mathrm{RN}-2$ & 35 & 12,15 & $\mathrm{R}$ \\
\hline C-RN-4 & 16 & 24,50 & MR & C-SE-2 & 36 & 11,86 & $\mathrm{R}$ \\
\hline C-MA-5 & 17 & 23,99 & MR & C-CE-2 & 37 & 10,98 & $\mathrm{R}$ \\
\hline C-CE-1 & 18 & 23,99 & MR & C-PE-2 & 38 & 10,94 & $\mathrm{R}$ \\
\hline C-PE-1 & 19 & 23,92 & MR & C-PI-1 & 39 & 10,73 & $\mathrm{R}$ \\
\hline C-RN-3 & 20 & 23,85 & MR & C-BA-2 & 40 & 10,56 & $\mathrm{R}$ \\
\hline
\end{tabular}

Ord.: ordenamento; R: resistente $(1,00 \leq \mathrm{IPD} \leq 12,99)$; $\mathrm{MR}$ : moderadamente resistente $(13,00 \leq \mathrm{IPD} \leq 25,99)$; e S:suscetível $(26,00 \leq \mathrm{IPD} \leq 50,00)$.

Os acessos C-RN-2, C-SE-2, C-CE-2, C-PE2, C-PI-1 e C-BA-2 com valores genotípicos de índice de porcentagem de doença menores do que 12,90 foram classificados como resistentes (Tabela 3), sendo apontados como fontes promissoras para serem empregadas em programas de melhoramento genético visando a incorporação de alelos de resistência a $P$. cubensis em cultivares de meloeiro. Vale salientar que o germoplasma avaliado no presente trabalho foi coletado em pequenas propriedades da agricultura tradicional do Nordeste brasileiro, com maior adaptação às condições edafoclimáticas regionais. No processo seletivo empregado pelos próprios agricultores é possível que tenha sido feita a seleção para $P$. cubensis na época chuvosa, mesmo que inconscientemente.

Considerando que o míldio é um dos principais problemas fitopatológicos do meloeiro na estação chuvosa acarretando a aplicação intensa de fungicidas por parte dos produtores, a identificação de novas fontes é um resultado relevante, uma vez que até os dias atuais são raros genótipos resistentes a $P$. cubensis. As fontes de resistência mais conhecidas, PI 124111, PI 124112 e MR-1 (THOMAS, 1982; 
THOMAS; JOURDAIN, 1992, THOMAS, 1986), possuem resistência parcial oligogênica (PERCHEPIED et al., 2005). Todas estas foram suscetíveis em ensaios anteriores em condições de campo na área experimental. Portanto, estes acessos podem ser apontados como fontes promissoras para serem empregadas em programas de melhoramento genético visando a incorporação de alelos de resistência a $P$. cubensis em cultivares de meloeiro.

A caracterização do germoplasma disponível quanto à reação aos patógenos de interesse é importante, pois estabelece um elo de ligação entre as informações disponíveis em bancos de germoplasma e o melhorista. Além disso, para o caso do meloeiro, é importante que as fontes de resistência identificadas sejam caracterizadas para variáveis qualitativas e quantitativas relacionados ao fruto, produto comercial desta cucurbitácea. Nesse sentido, realizou-se a caracterização das fontes de resistência promissoras e híbridos como referências (Tabela 4).

Os acessos resistentes diferiram quanto a coloração do epicarpo (amarela e creme) e mesocarpo (branca, verde e laranja), formato do fruto (alongado, oblongo, elíptico e esférico) e rachadura do fruto (presença e ausência) (Tabela 4), revelando a variabilidade morfológica dos frutos cultivados em pequenas propriedades do Nordeste brasileiro (TORRES et al., 2009). Por outro lado, todos os acessos foram monóicos e apresentaram desprendi- mento do pedúnculo dos frutos, um dos caracteres relacionados ao climatério no meloeiro (OBANDOULLOA et al., 2009).

Observou-se variação para o número de frutos por planta entre os acessos com maior destaque para C-PI-1 e C-BA-2. Espera-se obter maior número de frutos comerciais por plantas, uma vez que o número médio de frutos por planta na região Nordeste do Brasil é 1,5. Com relação à massa média observou-se uma amplitude de 0,5 a $3,7 \mathrm{~kg}$ (Tabela 4). O mercado externo prefere, principalmente, frutos menores que possam ser consumidos de uma só vez. Comercialmente, frutos com peso que variam de 1,0 a $1,5 \mathrm{~kg}$ são os preferidos para os tipos Amarelo, Honey Dew, Cantaloupe, Charenthais e Galia. Todavia, frutos de melão Pele de sapo com pesos superiores a $3,0 \mathrm{~kg}$ são bastante apreciados no mercado espanhol.

Considerando a espessura e a firmeza da polpa, os acessos podem ser caracterizados como de baixa qualidade de frutos, pois possuem reduzidos valores para esses caracteres quando comparados aos híbridos. A espessura da polpa é a parte comestível, sendo desejável a maior possível. A firmeza de polpa está relacionada com a vida útil pós-colheita e relaciona-se diretamente com as propriedades mecânicas de resistência fundamentais no manuseio, transporte, armazenamento e comercialização (BARROS et al., 2011).

Tabela 4. Caracterização morfológica de acessos de meloeiro coletados na região Nordeste do Brasil resistentes a P. cubensis em condições de campo.

\begin{tabular}{|c|c|c|c|c|c|c|c|c|c|}
\hline Acesso & $\mathrm{CE}$ & $\mathrm{CM}$ & $\mathrm{RF}$ & $\mathrm{FF}$ & $\mathrm{NF}$ & MF & EP & $\mathrm{FP}$ & SS \\
\hline 'Amaral' & Am & $\mathrm{Br}$ & Não & Obl & 1,3 & 1,75 & 4,3 & 28,12 & 10,5 \\
\hline 'Mabel' & Am & $\mathrm{Br}$ & Não & Obl & 1,4 & 1,90 & 4,5 & 29,32 & 9,8 \\
\hline 'Olimpic' & $\mathrm{Am}$ & $\mathrm{La}$ & Não & Esf & 1,2 & 1,45 & 4,6 & 22,15 & 12,4 \\
\hline $\mathrm{C}-\mathrm{RN}-2$ & $\mathrm{Cr}$ & $\mathrm{Br}$ & Sim & Alo & 1,9 & 1,50 & 2,9 & 18,10 & 4,3 \\
\hline C-SE-2 & Am & $\mathrm{Br}$ & Não & Esf & 1,0 & 3,70 & 3,0 & 15,20 & 5,7 \\
\hline C-CE-2 & $\mathrm{Cr}$ & $\mathrm{Ve}$ & Não & Eli & 1,1 & 1,80 & 2,6 & 15,50 & 4,6 \\
\hline C-PE-2 & $\mathrm{Am}$ & $\mathrm{La}$ & Não & Esf & 1,2 & 1,81 & 3,6 & 14,60 & 4,7 \\
\hline C-PI-1 & $\mathrm{Cr}$ & $\mathrm{Br}$ & Sim & Obl & 2,1 & 0,50 & 2,1 & 12,20 & 6,1 \\
\hline C-BA-2 & Am & $\mathrm{La}$ & Sim & Alo & 2,5 & 1,60 & 2,7 & 18,60 & 3,7 \\
\hline Média $_{(\mathrm{Ac})}$ & & & & & 1,3 & 1,70 & 4,5 & 26,53 & 10,9 \\
\hline Média $_{(\mathrm{Hib})}$ & & & & & 1,6 & 1,82 & 2,8 & 15,70 & 4,85 \\
\hline Amplitude & & & & & 1,5 & 3,20 & 1,5 & 6,40 & 2,40 \\
\hline
\end{tabular}

CE: cor do epicarpo; CM: cor do mesocarpo; RF: rachadura do fruto; FF: formado fruto; NF: número de frutos por planta; MF: massa do fruto, em kg; EP: espessura da polpa; FP: firmeza da polpa; SS: sólidos solúveis, em ${ }^{\circ}$ Brix. Ve: verde; Am: amarelo; Br: branco; Cr: creme; La: laranja; Amo: andromonóica; Mo: monóica; Obl: oblongo; Esf: esférico; Alo: alongado; e Eli: elíptico.

O teor de sólidos solúveis é um fator tradicionalmente usado para definir a qualidade do melão (NUNES et al., 2008). A qualidade dos frutos dos acessos é baixa, uma vez que os valores do teor de sólidos solúveis foram muito inferiores ao mínimo $\left(9,0^{\circ}\right.$ Brix $)$ exigido na comercialização com o mercado europeu. Este fato indica que os acessos são úteis apenas como doador de resistência a $P$. cubensis para materiais com alta qualidade de frutos, como os híbridos atualmente cultivados no Semiárido brasi- 
leiro voltados para a exportação.

Os acessos classificados como resistentes podem ser considerados como promissores para utilização em programas de melhoramento genético visando resistência a $P$. cubensis. Uma vez identificada fontes de resistência, a próxima etapa é entender o controle genético da resistência nestas fontes. O conhecimento da herança é importante porque norteia o melhorista no processo de transferência de alelos de resistência para genótipos comerciais. Por outro lado, como os acessos resistentes não têm qualidade comercial em seus frutos infere-se que o melhoramento é mais dificultado, pois será necessário recuperar o background do genitor suscetível com alta produtividade e frutos de elevada qualidade conforme a exigência dos consumidores do melão brasileiro no exterior

\section{CONCLUSÕES}

Os acessos C-RN-2, C-SE-2, C-CE-2, C-PE2, C-PI-1 e C-BA-2 apresentaram resistência a $P$. cubensis e foram promissores para uso em programas de melhoramento visando resistência ao míldio do meloeiro.

\section{AGRADECIMENTOS}

Os autores agradecem a CAPES pela bolsa de mestrado concedida à primeira autora e ao $\mathrm{CNPq}$ (Processo 552999/2011-5) pelo apoio financeiro com fim à realização desta pesquisa.

\section{REFERÊNCIAS}

BARROS, A. K. A. et al. Diallel analysis of yield and quality traits of melon fruits. Crop Breeding and Applied Biotechnology, Viçosa, v. 11, n. 4, p. 313-319, 2011.

CÂNDIDA, D. V. et al. Controle genético da murcha do fusário (Fusarium oxysporum) em feijoeiro comum. Tropical Plant Pathology, Brasília, v. 34, n. 6, p. 379-384, 2009.

CARDOSO, J. E.; SANTOS, A. A.; VIDAL, J. C. Efeito do míldio na concentração de sólidos solúveis em frutos de meloeiro. Fitopatologia Brasileira, Brasília, v. 27, n. 4, p. 378-383, 2002.

COHEN, R; SCHREIBER, S; NERSON, H. Response of Melofon Breeding Line to Powdery Mildew, Downy Mildew, Fusarium Wilt, and Sudden Wilt. Plant Disease, Saint Paul, v. 79, n. 6, p. 616619, 1995.
IPGRI. International Plant Genetic Resources Institute. Descriptors for melon (Cucumis melo L.). Rome: IPGRI. 2003. 65p. Disponível em: < http:// www.bioversityinternational.org/uploads/tx_news/ De-

scriptors for_melon_Cucumis_melo_L._906.pdf $>$ . Acessado em: 01 jan. 2015.

LEBEDA, A.; WIDRLECHNER, M. P.; URBAN, J. Individual and population aspects of interactions between cucurbits and Pseudoperonospora cubensis: pathotypes and races. In: HOLMES, G.J. (Ed.) Proceedings of Cucurbitaceae 2006. Raleigh, NC: Universal Press, 2006. p. 453-467.

McKINNEY, H. H. Influence of soil temperature and moisture on infection of wheat seedlings by Helminthosporium sativum. Journal of Agricultural Research, Washington, v. 26, n. 5, p. 195-218, 1923.

MICHEREFF, S. J. et al. Diagrammatic scale to assess downy mildew severity in melon. Horticultura Brasileira, Brasília, v. 27, n. 1, p. 76-79, 2009.

NEITZKE, R. S. et al. Caracterização morfológica e dissimilaridade genética entre variedades crioulas de melão. Horticultura Brasileira, Brasília, v. 27, n. 4, p. 534-538, 2009.

NUNES, G. H. S. et al. Desempenho de híbridos do grupo inodorus em Mossoró. Horticultura Brasileira, Brasília, v. 23, n. 1, p. 90-94, 2005.

NUNES, G. H. S. et al. Correlações entre características de meloeiro. Caatinga, Mossoró, v. 21, n. 1, p. 107-112, 2008

OBANDO-ULLOA, J. M. et al. Discrimination of climacteric and non-climacteric fruit at harvest and at senescence stage by quality traits. Journal Science Food Agriculture, Chichester, v. 89, n. 10, p. 1743-1753, 2009.

OLCZAK-WOLTMAN, H.; MARCINKOWSKA, J.; NIEMIROWICZ-SZCZYT, K. The genetic basis of resistance to downy mildew in Cucumis spp.latest developments and prospects. Journal of Applied Genetics, Olomouc, v. 51, n. 3, p. 249-255, 2011 .

PERCHEPIED, L. et al. Relationship between loci conferring downy mildew and powdery mildew resistance in melon assessed by quantitative trait loci mapping. Phytopathology, Saint Paul, v. 95, n. 5, p. 556-565, 2005.

PITRAT, M. Melon. In: PROHENS, J.; NUEZ, F. (Eds.). Handbook of plant breeding. New York, Springer, 2008. p. 283-315. 
QUEIROZ, M. A. Germplasm of Cucurbitaceae in Brazil. Crop Breeding and Applied Biotechnology, Viçosa, v. 4, n. 4, p. 377-383, 2004.

RESENDE, M. D. V. Genética biométrica e estatística no melhoramento de plantas perenes. Brasília, DF: Embrapa Informação Tecnológica, 2002. $975 \mathrm{p}$.

RESENDE, M. D. V.; DUARTE, J. B. Precisão e controle de qualidade em experimentos de avaliação de cultivares. Pesquisa Agropecuária Tropical, Brasília, v. 37, n. 3, p, 182-194, 2007.

ROSTOVZEV, S. I. Beitrage zur Kenntnis der Peronosporeen. Flora, Regensburg, v. 92, p. 405-430, 1903.

SANTOS, G. R. et al. Reaction of melon genotypes to the gummy stem blight and the downy mildew. Horticultura Brasileira, Brasília, v. 27, n. 2, p. 160$165,2009$.

THINES, M.; VOGLMAYR, H.; GÖKER, M. Taxonomy and Phylogeny of the Downy Mildews (Peronosporaceae) In: LAMOUR, K.; KAMOUN, S. (Ed.). Oomycete Genetics and Genomics: Diversity, Interactions, and Research Tools. New Jersey: Hoboken, 2009. v. 1, cap. 3, p. 47-75.

THOMAS, C. E. Resistance to downy mildew in Cucumis melo. Plant Disease, Saint Paul, v. 66, n. 6, p. 500-502, 1982.

THOMAS, C. E. Downy and powdery mildew resistant muskmelon breeding line MR-1. HortScience, Alexandria, v. 21, n. 2, p. 329, 1986

THOMAS, C. E.; JOURDAIN, E. L. Evaluation of Melon Germplasm for Resistance to Downy Mildew. HortScience, Alexandria, v. 27, n. 5, p. 434-436, 1992.

THOMAS, C. E. Additional Evaluations of Cucumis melo L. Germplasm for Resistance to Downy Mildew. HortScience, Alexandria, v. 34, n. 5, p. 920921, 1999.

TORRES FILHO, J. et al. Caracterização morfológica de acessos de meloeiro coletados no nordeste brasileiro. Caatinga, Mossoró, v.22, n. 3, p. 174- 181, 2009.

URBAN, J.; LEBEDA, A. Variation of fungicide resistance in Czech populations of Pseudoperonospora cubensis. Journal of Phytopathology, Berlin, v. 155 , n. 3 , p. $143-51,2007$.

VARGAS, P. F. et al. Avaliação de parentais e híbridos experimentais de melão rendilhado. Ciência e
Agrotecnologia, Berlin, v. 34, n. 5, p. 1102-1108, 2010 .

VENCOVSKY, R. Herança quantitative. In: PATERNIANI, E.; VIEGAS, G. P. (Ed.) Melhoramento e produção de milho. Campinas, SP: Fundação Cargill, 1987. v. 1, cap.5, p. 137-214. 